\title{
SEMILINEAR FOURTH ORDER BOUNDARY VALUE PROBLEMS
}

\section{GERHARD METZEN}

\begin{abstract}
We study a certain linear fourth order differential operator and show the existence of solutions to corresponding nonlinear problems. It will be shown that a maximum principle holds and that under certain conditions the linear operator has a positive principal eigenvalue with corresponding positive eigenfunction.
\end{abstract}

\section{INTRODUCTION}

Recently a number of papers have appeared dealing with the existence of solutions and, in some cases, with the uniqueness of solutions of fourth order boundary value problems arising from the study of elastic beams (see for example Aftabizadeh [1], Yang [12], Gupta [6], Agarwal [2], just to name a few). All of these papers treat what are called "nonresonance problems" near the first eigenvalue and most results are established for the boundary condition when the function and its second derivative vanish on the boundary.

In this paper we separate parts of the second derivative from the nonlinearity and make it part of the linear operator. Specifically, we consider boundary value problems of the form

$$
\left\{\begin{array}{l}
u^{\prime \prime \prime \prime}(t)+h(t) u^{\prime \prime}(t)+g\left(t, u(t), u^{\prime}(t)\right)=f(t), \quad t \in(0, \pi), \\
u(0)=u(\pi)=0=u^{\prime \prime}(0)=u^{\prime \prime}(\pi) .
\end{array}\right.
$$

The special case when $h(t)=-b \in \mathbf{R}, t \in(0, \pi)$, is of particular interest. The operator $u^{\prime \prime \prime \prime}-b u^{\prime \prime}$ occurs naturally in the context of the bending of a beam with axial load (see, for example, Hu [7], Washizu [11]). In terms of the deformation of beams, the boundary condition corresponds to a beam which is simply supported at both ends.

First we will study the linear problem $u^{\prime \prime \prime}+h u^{\prime \prime}$. A maximum principle will be established which, in turn, will be used to prove further properties of the principal eigenvalue. In particular, we will show that under certain assumptions on $h$ we have a positive operator.

Finally, we will prove some existence theorems, based in part on the results of the previous section. These results complement those of the papers cited above and, in some cases, extend them.

Received 14 September 1989

Copyright Clearance Centre, Inc. Serial-fee code: 0004-9729/90 \$A2.00+0.00. 
Let us make a final remark about our notation. $C^{k}([a, b])$ denotes the Banach space of $k$-times continuously differentiable functions with norm

$$
|f|_{k}:=\sup _{\substack{0 \leqslant i \leqslant k \\ t \in[a, b]}}\left|f^{(i)}(t)\right|
$$

For $p \in[1, \infty], L^{p}(a, b)$ denotes the usual Lebesgue space with norm $\|\cdot\|_{p}$. However, the norm of the Hilbert space $L^{2}(a, b)$ is denoted simply by $\|\cdot\|$. For $p \in[1, \infty)$ and $k \in N$, we let $W^{k, p}(a, b)$ denote the Sobolev space

$$
W^{k, p}(a, b):=\left\{u \in L^{p}(a, b) \mid u^{(i)} \in L^{p}(a, b), i=1,2, \ldots, k\right\}
$$

with norm

$$
\|u\|_{k, p}:=\left(\sum_{i=0}^{k}\left(\left\|u^{(i)}\right\|_{p}\right)^{p}\right)^{1 / p}
$$

where, of course, $u^{(i)}$ denotes the $i$ th distributional derivative of $u$. If $p=2$, we set

$$
H^{k}(a, b):=W^{k, 2}(a, b)
$$

with norm $\|\cdot\|_{k, 2}$.

2. The Linear PROBLEM

For a function $u \in W^{2, p}(0, \pi)$ let $B_{1}(u):=u|\partial(0, \pi)=u|\{0, \pi\}$, and for $v \in W^{4, p}(0, \pi)$ we let $B_{2}(v):=v^{\prime \prime}\left|\partial(0, \pi)=v^{\prime \prime}\right|\{0, \pi\}$.

In this section we will prove the existence of a principal eigenvalue of the linear problem. To do this, we will first prove a version of the maximum principle.

LEMMA 1. Assume that a function $h:[0, \pi] \rightarrow R$ satisfies $h(t) \leqslant 0$ on $[0, \pi]$ and is bounded on $[0, \pi]$. Suppose that $u(t)$ satisfies

$$
\begin{cases}u^{\prime \prime \prime \prime}(t)+h(t) u^{\prime \prime}(t) \geqslant 0, & t \in(0, \pi) \\ B_{1} u=0, \quad u^{\prime \prime}(0) \leqslant 0, & u^{\prime \prime}(\pi) \leqslant 0 .\end{cases}
$$

If $u^{\prime \prime}(t)$ is continuous on $[0, \pi]$, then either

$$
u(t)=0 \text { in }[0, \pi] \text { or } u(t)>0 \text { in }(0, \pi)
$$

Proof: Set $v(t):=u^{\prime \prime}(t)$. Then $v(t)$ satisfies

$$
\left\{\begin{array}{l}
v^{\prime \prime}(t)+h(t) v(t) \geqslant 0, \quad t \in(0, \pi) \\
v(0) \leqslant 0, \quad v(\pi) \leqslant 0 .
\end{array}\right.
$$


Applying the standard maximum principle to this (see Protter-Weinberger [9]), we deduce that either

$$
v(t)=0 \text { in }[0, \pi] \quad \text { or } \quad v(t)<0 \text { in }(0, \pi) .
$$

Now, if $v(t)=0$ in $[0, \pi]$, then $u^{\prime \prime}(t)=0$ in $[0, \pi]$ and $B_{1} u=0$. This clearly implies that $u(t)=0$ in $[0, \pi]$.

If, on the other hand, $v(t)<0$ in $(0, \pi)$, then $u(t)$ satisfies

$$
\left\{\begin{array}{l}
u^{\prime \prime}(t)<0, \\
u(0)=0=u(\pi)
\end{array} \quad t \in(0, \pi),\right.
$$

Again, by the standard maximum principle we obtain $u(t)>0$ in $(0, \pi)$. This proves the lemma.

REMARK 1. If one of the inequalities in (2) does not reduce to an equality, then, of course, the conclusion of Lemma 1 is that $u(t)>0$ in $(0, \pi)$.

The next result is a boundary point lemma.

Lemma 2. Let $h$ be as in Lemma 1 and assume that $u \in W^{4,1}(0, \pi)$ satisfies $u(t)>0$ in $(0, \pi)$ and

$$
\left\{\begin{array}{l}
u^{\prime \prime \prime \prime}(t)+h(t) u^{\prime \prime}(t) \geqslant 0, \quad t \in(0, \pi), \\
B_{1} u=0=B_{2} u .
\end{array}\right.
$$

Then $u^{\prime}(0)>0$ and $u^{\prime}(\pi)<0$.

Proof: Again, we set $v(t):=u^{\prime \prime}(t)$. Then

$$
\left\{\begin{array}{l}
v^{\prime \prime}(t)+h(t) v(t) \geqslant 0, \quad t \in(0, \pi), \\
v(0)=0=v(\pi)
\end{array}\right.
$$

implying that either $v(t)=0$ in $[0, \pi]$ or $v(t)<0$ in $(0, \pi)$. However, since $u(t)>0$ in $(0, \pi)$ and $B_{1} u=0$, we must conclude that

$$
v(t)=u^{\prime \prime}(t)<0 \text { in }(0, \pi) .
$$

Now, for each $r \in(0, \pi)$ there exists some $s(r) \in(0, r)$ such that

$$
u(r)=u(0)+u^{\prime}(0) r+u^{\prime \prime}(s(r)) r^{2} / 2 .
$$

Since $u(0)=0$, we actually have

$$
u(r)=u^{\prime}(0) r+u^{\prime \prime}(s(r)) r^{2} / 2
$$


This shows that we cannot have $u^{\prime}(0)<0$. Suppose we had $u^{\prime}(0)=0$. Then

$$
u(r)=u^{\prime \prime}(s(r)) r^{2} / 2 .
$$

Now $s(r) \in(0, r) \subseteq(0, \pi)$ and therefore $u^{\prime \prime}(s(r))<0$. This, again, contradicts our assumption that $u(t)>0$ in $(0, \pi)$. We therefore must have $u^{\prime}(0)>0$.

The argument that $u^{\prime}(\pi)<0$ is similar. In this case we merely use the fact that

$$
u(r)=u(\pi)+u^{\prime}(\pi)(r-\pi)+u^{\prime \prime}(t(r))(r-\pi)^{2} / 2
$$

for some $t(r) \in(r, \pi)$.

Next, we will study the operator

$$
L_{h} u:=u^{\prime \prime \prime \prime}+h(t) u^{\prime \prime},
$$

where $\operatorname{dom}\left(L_{h}\right):=\left\{u \in H^{4}(0, \pi) \mid B_{1} u=0=B_{2} u\right\}$ and where $h \in L^{\infty}(0, \pi)$ satisfies $h(t) \leqslant 0$ in $[0, \pi]$.

Let $R(f):=v$, where $v$ is the unique solution of problem

$$
\left\{\begin{array}{l}
v^{\prime \prime}(t)=h(t) v(t)=f(t), \quad t \in(0, \pi), \\
B_{1} v=0 .
\end{array}\right.
$$

and set $S(f):=w$, where $w$ is the unique solution of the problem

$$
\left\{\begin{array}{l}
w^{\prime \prime}(t)=f(t), \quad t \in(0, \pi), \\
B_{1} w=0
\end{array}\right.
$$

Then $R$ and $S$ are the solution operators corresponding to those two problems, and it is well-known that $R, S: L^{2}(0, \pi) \rightarrow C^{1}([0, \pi])$ are compact linear operators. Also note that

$$
L_{h} u=f \Longleftrightarrow u=(S \circ R)(f)
$$

Using regularity theory, we see that $T:=S \circ R$ is a compact linear operator from $L^{2}(0, \pi)$ into $C^{3}([0, \pi])$.

Our goal is to show that $T$ is a positive operator on some appropriate ordered Banach space. To this end, we set $X:=C_{0}([0, \pi]):=\left\{u \in C([0, \pi]) \mid B_{1} u=0\right\}$ with positive cone

$$
K:=\left\{u \in C_{0}([0, \pi]) \mid u(t) \geqslant 0, \quad t \in[0, \pi]\right\} .
$$

Since $\operatorname{int}(K)=\emptyset$, this setup is unsuitable. We therefore resort to a different Banach space in the following manner. Set $e(t):=\sin t, t \in[0, \pi]$, and define $X_{e}$ and $\|\cdot\|_{e}$ by

$$
X_{e}:=\left\{u \in C_{0}([0, \pi]) \mid \exists a>0 \text { such that }|u(t)|<a e(t), t \in[0, \pi]\right\}
$$


and

$$
\|u\|_{e}:=\inf \{a>0|| u(t) \mid \leqslant a e(t), \quad t \in[0, \pi]\}
$$

We also set

$$
K_{e}:=K \cap X_{e} .
$$

It is well-known that $\left(X_{e},\|\cdot\|_{e}\right)$ is a Banach space with normal order cone $K_{e}$ and $\operatorname{int}\left(K_{e}\right) \neq \emptyset$. For details see Amann [3], Deimling [5], Zeidler [13].

Lemмa 3. The embedding $\left(C_{0}^{1}([0, \pi]),|\cdot|_{1}\right) \rightarrow\left(X_{e},\|\cdot\|_{e}\right)$ is continuous.

Proof: Note that $2 e(t)>1$ on the interval $I_{1}:=[1 / \sqrt{2}, \pi-1 / \sqrt{2}], 2 e^{\prime}(t)>1$ on $I_{2}:=[0,(1 / \sqrt{2})]$ and $2 e^{\prime}(t)<-1$ on $I_{3}:=[\pi-1 / \sqrt{2}, \pi]$. The first statement implies that $|u(t)| \leqslant 2|u|_{1} e(t)$ on $I_{1}$, while the second and third statement imply that the same inequality also holds on $I_{2}$ and $I_{3}$, respectively. Therefore

$$
|u(t)| \leqslant 2|u|_{1} e(t), \quad t \in[0, \pi]
$$

which implies that

$$
\|u\|_{e} \leqslant 2|u|_{1} .
$$

This shows that the embedding is bounded (see Deimling [5]).

It is also easily seen that the embedding $\left.\left(X_{e},\|\cdot\|_{e}\right) \rightarrow\left(C_{0}(0, \pi]\right), H_{0}\right)$ is continuous. Now set $T_{e}:=T \mid X_{e}$ and $T_{0}:=T \mid C_{0}([0, \pi])$. Then $T_{e}: X_{e} \rightarrow X_{e}$ and $T_{0}:\left(C_{0}([0, \pi]),|\cdot|_{0}\right) \rightarrow\left(C_{0}^{1}([0, \pi]),|\cdot|_{1}\right)$ are compact. Since the linear operator $T_{c}$ has the following factorisation

$$
\left(X_{e},\|\cdot\|_{e}\right) \rightarrow\left(C_{0}([0, \pi]),|\cdot|_{0}\right) \stackrel{T_{0}}{\longrightarrow}\left(C_{0}^{1}([0, \pi]),|\cdot|_{1}\right) \rightarrow\left(X_{e},\|\cdot\|_{e}\right),
$$

it follows that $T_{e}: X_{e} \rightarrow X_{e}$ is compact.

Lemma 4. We have $T_{e}\left(K_{e} \backslash\{0\}\right) \subseteq \operatorname{int}\left(K_{e}\right)$.

Proof: Observe that $u \in C_{0}([0, \pi])$ belongs to int $\left(K_{e}\right)$ if and only if $u(t)>0$ in $(0, \pi)$ and $u^{\prime}(\pi)<0<u^{\prime}(0)$. Now, if $f \in K_{e} \backslash\{0\}$, then by Lemma 1 we conclude that $u:=T_{e}(f)$ satisfies $u(t)>0$ in $(0, \pi)$ and hence Lemma 2 implies that $u^{\prime}(\pi)<$ $0<u^{\prime}(0)$. Therefore $u \in \operatorname{int}\left(K_{e}\right)$.

THEOREM 1. The eigenvalue problem

$$
L_{h} u=\lambda u
$$

has a simple real eigenvalue $\lambda_{1}>0$ and the corresponding eigenfunction $\varphi \in \operatorname{int}\left(K_{e}\right)$. Any other eigenvalue $\lambda$ of $L_{h}$ satisfies $\lambda_{1}<|\lambda|$ and any eigenfunction corresponding to $\lambda$ must change sign.

Proof: We note that $0 \notin \sigma(L)$ and therefore $\lambda \in \sigma(L)$ if and only if $1 / \lambda \in \sigma\left(T_{e}\right)$. Since, by Lemma $4, T_{e}$ is strongly positive, we see that we can apply the Krein-Rutman 
theorem (see Zeidler [13], Amann [3], Deimling [5]). From this, Theorem 1 follows immediately.

Let us assume now that $h \in C^{2}([0, \pi])$ and let $u, v \in \operatorname{dom}\left(L_{h}\right)$. A simple calculation shows that

$$
\left\langle L_{h} u, v\right\rangle=\left\langle u, v^{\prime \prime \prime \prime}+(h v)^{\prime \prime}\right\rangle .
$$

Therefore $\operatorname{dom}\left(L_{h}\right) \subseteq \operatorname{dom}\left(L_{h}^{*}\right)$. In fact, the following is true.

Lemma 5. If $h \in C^{2}([0, \pi])$, then $L_{h}^{*}: \operatorname{dom}\left(L_{h}^{*}\right) \subseteq L^{2}(0, \pi) \rightarrow L^{2}(0, \pi)$ is given by

$$
L_{h}^{*}(v)=v^{\prime \prime \prime \prime}+(h v)^{\prime \prime}
$$

where $\operatorname{dom}\left(L_{h}^{*}\right)=\left\{v \in H^{4}(0, \pi) \mid B_{1} v=0=B_{2} v\right\}$.

Proof: Let $v \in \operatorname{dom}\left(L_{h}^{*}\right) \subseteq L^{2}(0, \pi)$. Then $v^{\prime \prime \prime \prime}+(h v)^{\prime \prime}$ is a distribution and therefore

$$
\left[v^{\prime \prime \prime \prime}+(h v)^{\prime \prime}\right](f)=v\left(f^{\prime \prime \prime \prime}\right)+v\left(h f^{\prime \prime}\right)=\left\langle v, L_{h} f\right\rangle=\left\langle L_{h}^{*} v, f\right\rangle
$$

for all $f \in C_{0}^{\infty}(0, \pi)$. This implies that we in fact have

$$
L_{h}^{*} v=v^{\prime \prime \prime \prime}+(h v)^{\prime \prime}
$$

In particular, $L_{h}^{*} v \in L^{2}(0, \pi)$ implies, by regularity, that $v \in H^{4}(0, \pi)$. Hence $\operatorname{dom}\left(L_{h}^{*}\right) \subseteq H^{4}(0, \pi)$. Now, if $u \in \operatorname{dom}\left(L_{h}\right)$ and $v \in \operatorname{dom}\left(L_{h}^{*}\right)$, then

$$
\left\langle L_{h} u, v\right\rangle=\left\langle u, L_{h}^{*} v\right\rangle .
$$

Using integration by parts, we find that the left-hand side is given by

$$
\left\langle L_{h} u, v\right\rangle=\left.\left(u^{\prime \prime \prime} v+u^{\prime} v^{\prime \prime}+u^{\prime} h v\right)\right|_{0} ^{\pi}+\left\langle u, L_{h}^{*} v\right\rangle .
$$

Therefore

$$
\left.\left(u^{\prime \prime \prime} v+u^{\prime} v^{\prime \prime}+u^{\prime} h v\right)\right|_{0} ^{\pi}=0
$$

for all $u \in \operatorname{dom}\left(L_{h}\right)$. It is now easily seen, by constructing appropriate functions $u \in \operatorname{dom}\left(L_{h}\right)$, that $B_{1} v=0=B_{2} v$. This shows that $\operatorname{dom}\left(L_{h}^{*}\right)=\operatorname{dom}\left(L_{h}\right)$ as claimed.

Next, let $h \in C^{2}([0, \pi])$ satisfy $h(t) \leqslant 0$ on $[0, \pi]$. A further consequence of the Krein-Rutman theorem is that $1 / \lambda_{1}$ is also a simple eigenvalue of $T_{e}^{*}$ with corresponding strictly positive eigenfunction $\xi \in X_{e}^{*}$. Note also that $\lambda_{1}$ is an eigenvalue of $L_{h}^{*}$ with corresponding eigenfunction $\theta \in \operatorname{dom}\left(L_{h}^{*}\right)$ and that $\operatorname{dom}\left(L_{k}^{*}\right) \subseteq X_{e}^{*}$.

Now let $w \in X_{e}$ and set $u:=T_{e} w$. Then

$$
\begin{aligned}
\langle w, \theta\rangle & =\left\langle L_{h} u, \theta\right\rangle=\left\langle u, L_{h}^{*} \theta\right\rangle=\left\langle T_{e} w, \lambda_{1} \theta\right\rangle=\left\langle\lambda_{1} T_{e} w, \theta\right\rangle \\
& =\lambda_{1} T_{e}^{*} \theta(w) .
\end{aligned}
$$


Therefore $T_{e}^{*} \theta=1 / \lambda_{1} \theta$ and so $\theta$ is strictly positive, that is,

$$
\langle\theta, u\rangle>0 \text { for all } u \in K_{\mathrm{e}} \backslash\{0\}
$$

This clearly implies that $\theta(t)>0$ for all $t \in(0, \pi)$. Summarising, we have proved the following theorem.

THEOREM 2. Let $h \in C^{2}([0, \pi])$ satisfy $h(t) \leqslant 0$ for all $t \in[0, \pi]$. Then the eigenvalue problem

$$
L_{h}^{*} u=\lambda u
$$

has the simple eigenvalue $\lambda_{1}$ (the principle eigenvalue of $L_{h}$ ) and a corresponding eigenfunction $\theta \in \operatorname{dom}\left(L_{h}^{*}\right)$ such that $\theta(t)>0$ for all $t \in(0, \pi)$.

If the function $h$ is constant, that is, if $h(t)=-b, t \in[0, \pi]$, for some constant $b \in \mathbf{R}^{+}$, then of course $\sigma\left(L_{k}\right)$ is easily calculated. In fact, in this case we have

$$
\sigma\left(L_{h}\right)=\left\{n^{4}+n^{2} b \mid n \in N\right\} .
$$

It is very useful to know what happens to the principal eigenvalue as the function $h(t)$ changes. In other words, assume that $h, p \in L^{\infty}(0, \pi)$ satisfy $h(t) \leqslant p(t) \leqslant 0$ and let $\mu_{1}$ denote the principal eigenvalue of $L_{p}$. In the next theorem we will show that $\mu_{1} \leqslant \lambda_{1}$.

THEOREM 3. Let $\lambda_{1}$ and $\mu_{1}$ denote the principal eigenvalues of $L_{h}$ and $L_{p}$, respectively. Here $h, p \in L^{\infty}(0, \pi)$ are such that $h(t) \lessgtr p(t) \leqslant 0$. Then

$$
0<\mu_{1}<\lambda_{1} \text {. }
$$

Proof: Let $T_{e}, P_{e}: X_{e} \rightarrow X_{e}$ denote the restriction of the solution operator of $L_{k}$ and $L_{p}$, respectively. Now let $w \in K_{e} \backslash\{0\}$ and set $u:=T_{e}(w)$ and $v:=P_{e}(w)$. Then $u$ and $v$ satisfy the homogeneous boundary conditions and the respective differential equations

$$
u^{\prime \prime \prime \prime}+h u^{\prime \prime}=\boldsymbol{w}
$$

and

$$
v^{\prime \prime \prime \prime}+p v^{\prime \prime}=w
$$

Therefore

$$
(u-v)^{\prime \prime \prime \prime}+h u^{\prime \prime}-p v^{\prime \prime}=0
$$


Applying the maximum principle (Lemma 1 ) to (8), we conclude that $v^{\prime \prime}<0$ in $(0, \pi)$ and hence $-h v^{\prime \prime} \lesseqgtr-p v^{\prime \prime}$. Now we have

$$
(u-v)^{\prime \prime \prime \prime}+h(u-v)^{\prime \prime} \lesseqgtr 0 .
$$

Since $(u-v)$ also satisfies the homogeneous boundary conditions, we must have $(u-v)<0$ in $(0, \pi)$ and therefore $u<v$ in $(0, \pi)$. We have shown that $w>0$ implies $T_{\mathrm{e}}(w)<P_{\mathrm{e}}(w)$. Now we deduce from Corollary 7.28 in Zeidler [13] that the spectral radius of $T_{e}$ is less than that of $P_{e}$, that is, $1 / \lambda_{1}<1 / \mu_{1}$. Therefore $\mu_{1}<\lambda_{1}$.

Assume that $b \in \mathbf{R}^{+}$. Then the principal eigenvalue of

$$
\left\{\begin{array}{l}
u^{\prime \prime \prime \prime}-b u "=\lambda_{1} u \\
B_{1} u=0=B_{2} u
\end{array}\right.
$$

is $\lambda_{1}=1+b$. If now $h \in L^{\infty}(0, \pi)$ is such that $b \lesseqgtr h(t), t \in[0, \pi]$, and if $\mu_{1}$ denotes the principal eigengalue of

$$
\left\{\begin{array}{l}
u^{\prime \prime \prime \prime}-h(t) u^{\prime \prime}=\mu u \\
B_{1} u=0=B_{2} u,
\end{array}\right.
$$

then it follows from the above discussion that $1+b<\mu_{1}$.

Let again $h \in L^{\infty}(0, \pi)$ be such that $h(t) \leqslant 0$ on $[0, \pi]$. We know that if $h$ is a constant, then $L_{h}$ is a positive operator, that is,

$$
\left\langle L_{h} u, u\right\rangle>0 \text { for all } u \in \operatorname{dom}\left(L_{h}\right) \backslash\{0\} .
$$

One may wonder whether the same is true if $h$ is not a constant. Below, we will give an example showing that $L_{h}$ is not necessarily positive for any $h \in L^{\infty}(0, \pi)$ with $h(t) \leqslant 0$.

EXAMPLE. Let $h \in L^{\infty}(0, \pi)$ be defined by

$$
h(t):= \begin{cases}-1000 \pi, & \text { if } t \in I, \\ 0, & \text { otherwise }\end{cases}
$$

where $I:=[0.4,0.6] \cup[\pi-0.6, \pi-0.4]$. Also set

$$
u(t):=[t(t-\pi)]^{3}, \quad t \in[0, \pi] .
$$

Then $u \in \operatorname{dom}\left(L_{h}\right)$ and a simple, but tedious, calculation shows that

$$
\left\langle L_{h} u, u\right\rangle<0 .
$$

(Note that $\left\|u^{\prime \prime}\right\|_{\infty}<40$ and on $I$ we have $u(t)<-1$ and $u^{\prime \prime}(t)<-20$.)

However, if we impose some conditions on $h(t)$, then $L_{h}$ will be a positive operator. 
THEOREM 4. Let $h \in H^{2}(0, \pi)$ and $b \in \mathbf{R}$ be such that $h(t) \leqslant-b, t \in[0, \pi]$. Then

$$
\left\langle L_{h} u, u\right\rangle \geqslant\left(1+b-\left\|h^{\prime}\right\|_{\infty}\right)\left\|u^{\prime}\right\|^{2} .
$$

Proof: Note that for $u \in \operatorname{dom}\left(L_{h}\right)$ we have

$$
\|u\| \leqslant\left\|u^{\prime}\right\| \text { and }\left\|u^{\prime}\right\| \leqslant\left\|u^{\prime \prime}\right\| \text {, }
$$

and for every $f \in L^{2}(0, \pi)$ we have

$$
\|f\|^{2} \leqslant \sqrt{\pi}\left\|f^{2}\right\|
$$

Now

$$
\begin{aligned}
\langle L u, u\rangle & =\int_{0}^{\pi}\left(u^{\prime \prime}\right)^{2} d t-\int_{0}^{\pi} h\left(u^{\prime}\right)^{2} d t-\int_{0}^{\pi} h^{\prime} u u^{\prime} d t \\
& \geqslant\left\|u^{\prime \prime}\right\|^{2}+b \int_{0}^{\pi}\left(u^{\prime}\right)^{2} d t-\left\|h^{\prime}\right\|_{\infty}\|u\|\left\|u^{\prime}\right\| \\
& \geqslant\left\|u^{\prime \prime}\right\|^{2}+b\left\|u^{\prime}\right\|^{2}-\left\|h^{\prime}\right\|_{\infty}\left\|u^{\prime}\right\|^{2} \\
& \geqslant\left(1+b-\left\|h^{\prime}\right\|_{\infty}\right)\left\|u^{\prime}\right\|^{2} .
\end{aligned}
$$

This proves the theorem.

If $h$ is constant, it is clear that the operator $L_{h}$ is self-adjoint. As one might suspect, this is the only case when $L_{h}$ is self-adjoint.

THEOREM 5. Let $h \in L^{\infty}(0, \pi)$. Then $L_{h}$ is self-adjoint if and only if $h$ is constant almost everywhere.

Proof: It suffices to show that the self-adjointness of $L_{h}$ implies that $h$ is constant almost every where. To that end, we assume that $L_{h}$ is self-adjoint. Then we certainly must have $\left\langle L_{h} u, v\right\rangle=\left\langle u, L_{h} u\right\rangle$ for all $u, v \in \operatorname{dom}\left(L_{h}\right)$ and therefore

$$
\int_{0}^{\pi} h\left[u^{\prime \prime} v-u v^{\prime \prime}\right] d t=0, \quad \text { for all } u, v \in \operatorname{dom}\left(L_{h}\right)
$$

Let $g$ be an arbitrary function in $B_{0}^{1}(0, \pi)$. Then there exists a sequence $\left\{g_{k}\right\} \subseteq$ $C_{0}^{\infty}(0, \pi)$ such that

$$
g_{k} \rightarrow g \text { in } H_{0}^{1}(0, \pi) \text { as } k \rightarrow \infty .
$$

Fix $k \in N$ and set $v(t):=\sin t$ and

$$
u(t):=\sin t \int_{0}^{t} \frac{g_{k}(s)}{\sin ^{2} s} d s .
$$


It then follows that $u, v \in \operatorname{dom}\left(L_{h}\right)$ and

$$
u^{\prime \prime} v-u v^{\prime \prime}=g_{k}^{\prime} \text { in }[0, \pi] \text {. }
$$

Therefore

$$
\int_{0}^{\pi} h(t) g_{k}^{\prime}(t) d t=0
$$

for all $k \in N$. From this we deduce that

$$
\int_{0}^{\pi} h(t) g^{\prime}(t) d t=0
$$

for all $g \in H_{0}^{1}(0, \pi)$. Finally, by the lemma of Du Bois Raymond from the calculus of variations we conclude that $h$ is constant almost everywhere.

In the next section we will show how some of the results from this section can be used to extend, at least in some special cases, certain types of existence results.

\section{NONLINEAR PROBLEMS}

In this section we will prove the existence of solutions of certain nonlinear problems. Our results compliment those of Gupta $[6]$ and, in some special cases, improve them.

Many of the existence results (and also multiplicity results) which are known for elliptic boundary value problems can be carried over to problems such as (1). This is because one often makes use only of certain abstract properties of the elliptic problems. The case when $h(t) \leqslant 0, t \in[0, \pi]$, is particularly nice because in this case $L_{h}$ has a positive principal eigenvalue with corresponding positive eigenfunction $\varphi$ satisfying $\varphi^{\prime}(\pi)<0<\varphi^{\prime}(0)$ (see Theorem 1). This is very useful in many cases.

Below we will give an example of one such result for a so-called resonance problem.

THEOREM 6. Let $h(t):=-b \in \mathbf{R}$ for all $t \in[0, \pi]$ and assume that $g:[0, \pi] \times \mathbf{R} \rightarrow$ $\mathbf{R}$ is a Carathéodory function satisfying:

(i) for every $R>0$ there exists a function $f_{R} \in L^{1}(0, \pi)$ such that

$$
|g(t, s)| \leqslant f_{R}(t)
$$

for all $t \in[0, \pi]$ and all $s \in \mathbf{R}$ with $|s| \leqslant R$;

(ii) there exist functions $c, d \in L^{\infty}(0, \pi)$ such that

$$
\begin{aligned}
& -1-b \leqslant \liminf _{s \rightarrow+\infty} \frac{g(t, s)}{s} \leqslant \limsup _{s \rightarrow+\infty} \frac{g(t, s)}{s} \leqslant c(t) \\
& -1-b \leqslant \liminf _{s \rightarrow-\infty} \frac{g(t, s)}{s} \leqslant \limsup _{s \rightarrow-\infty} \frac{g(t, s)}{s} \leqslant d(t)
\end{aligned}
$$

uniformly almost everywhere in $t \in(0, \pi)$. 
Let $f \in L^{1}(0, \pi)$ be such that

$$
\int_{0}^{\pi} g(t,-\infty) \sin t d t<\int_{0}^{\pi} f(t) \sin t d t<\int_{0}^{\pi} g(t,+\infty) \sin t d t
$$

where $g(t,-\infty):=\limsup _{s \rightarrow-\infty}[g(t, s)+(1+b) s]$ and $g(t,+\infty):=\liminf _{s \rightarrow+\infty}[g(t, s)+(1+b) s]$. Then the problem

$$
\left\{\begin{array}{l}
u^{\prime \prime \prime \prime}(t)-b u^{\prime \prime}(t)+g(t, u(t))=f(t), \quad t \in(0, \pi), \\
B_{1} u=0=B_{2} u
\end{array}\right.
$$

has a solution $u \in W^{4,1}(0, \pi)$.

Proof: Since $h(t)$ is constant, it follows that $L_{h}$ is self-adjoint. A simple application of spectral analysis shows that if $u \in \operatorname{dom}\left(L_{h}\right)$ is a solution of

$$
L_{h} u+\mu u^{+}-\nu u^{-}=0,
$$

where $\mu, \nu \in L^{\infty}(0, \pi)$ are such that $(\mu(t), \nu(t)) \in[-1-b, c(t)] \times[-1-b, d(t)]$ for almost everywhere $t \in(0, \pi)$, then $u \in \operatorname{ker}\left(L_{h}-(1+b)\right)$. Using this and the fact that the differential operator $L_{h}$ gives rise to a differential operator in $L^{1}(0, \pi)$, that is,

$$
L: \operatorname{dom}(L) \subseteq L^{1}(0, \pi) \rightarrow L^{1}(0, \pi) .
$$

This operator has a compact resolvent as a map from $L^{1}(0, \pi)$ into $\left(C^{-1}([0, \pi]),|\cdot|_{1}\right)$. Actually, we have more regularity (see Section 2), but this is all we need. We now can apply Theorem 3 in $[8]$ to obtain a solution.

Remark 2. (a) Theorem 6 also holds true for an arbitrary function $h \in L^{\infty}(0, \pi)$ if it can be shown that a solution $u \in \operatorname{dom}\left(L_{h}\right)$ of (10) must lie in $\operatorname{ker}\left(L_{h}-\lambda_{1}\right)$. Of course, $1+b$ must be replaced by $\lambda_{1}$ and $\sin t$ by a positive eigenfunction $\theta$ corresponding to the principal eigenvalue $\lambda_{1}$. Again, this follows from a simple application of Theorem 3 in $[8]$.

(b) Similar results also hold for higher eigenvalues. One can directly apply Theorem 2 of [8] or use the results and ideas of [4].

Our next theorem is a so-called nonresonance result. Here we allow $h$ to be nonconstant but assume some regularity on $h$.

THEOREM 7. Let $h \in H^{2}(0, \pi)$ and $b \in \mathbf{R}^{+}$be such that $h(t) \leqslant-b$ for all $t \in[0, \pi]$. Suppose $g:[0, \pi] \times \mathbf{R}^{2} \rightarrow \mathbf{R}$ is a Carathéodory function satisfying:

(i) for some $a_{1}, a_{2}, a_{3}, a_{4} \in L^{\infty}(0, \pi)$ we have

$$
g(t, s, r) s \geqslant a_{1}(t) s^{2}+a_{2}(t) s r+a_{3}(t) s+a_{4}(t) r
$$


for all $(t, s, r) \in[0, \pi] \times R^{2}$;

(ii) there exist constants $b_{1}, b_{2} \in \mathrm{R}^{+}$and a function $b_{0} \in L^{1}(0, \pi)$ such that

$$
|g(t, s, r)| \leqslant b_{0}(t)+b_{1}|s|+b_{2}|r|
$$

for all $(t, r, s) \in[0, \pi] \times R^{2}$.

If the condition

$$
-\frac{\pi^{2}}{4} \int_{0}^{\pi} a_{1}^{-}(t) d t>-1-b+\left\|h^{\prime}\right\|_{\infty}+\frac{\pi}{2}\left\|a_{2}\right\|
$$

holds, where $a_{1}^{-}(t):=\min \left\{a_{1}(t), 0\right\}$, then for every $f \in L^{1}(0, \pi)$, the problem

$$
\left\{\begin{array}{l}
u^{\prime \prime \prime \prime}(t)+h(t) u^{\prime \prime}(t)+g\left(t, u(t), u^{\prime}(t)\right)=f(t), \quad t \in(0, \pi) \\
B_{1} u=0=B_{2} u
\end{array}\right.
$$

has a solution $u \in W^{4,1}(0, \pi)$.

Proof: Again, let $L: \operatorname{dom}(L) \subseteq L^{1}(0, \pi) \rightarrow L^{1}(0, \pi)$ denote the differential operator corresponding to (12) (clearly $L_{h} \subseteq L$ ). Let $N: H^{1}(0, \pi) \rightarrow L^{1}(0, \pi)$ be defined by

$$
(N u)(t):=f(t)-g\left(t, u(t), u^{\prime}(t)\right), \quad t \in(0, \pi) .
$$

We then employ the Leray-Schauder degree to solve the problem

$$
L u=N u
$$

which is equivalent to (12). To this end, we consider the homotopy

$$
L u=r N u, \quad r \in[0,1]
$$

First we shall show that the set of all solutions to (13) is bounded with respect to the $\|\cdot\|_{2,2}$-norm.

Now

$$
\langle L u, u\rangle=r\langle N u, u\rangle
$$

and from Theorem 4 we have

$$
\langle L u, u\rangle \geqslant \pi^{-1 / 2}\left(1+b-\sqrt{\pi}\left\|h^{\prime}\right\|_{\infty}\right)\left\|u^{\prime}\right\|^{2} \text {. }
$$

Again, using $\|u\| \leqslant\left\|u^{\prime}\right\|,\|u\|^{2} \leqslant \sqrt{\pi}\left\|u^{2}\right\|$ and condition (i) above, we obtain

$$
r\langle N u, u\rangle \leqslant \frac{\pi^{2}}{4}\left\|u^{\prime}\right\|^{2} \int_{0}^{\pi} a_{1}^{-}(t) d t+\frac{\pi}{2}\left\|a_{2}\right\|\left\|u^{\prime}\right\|^{2}+l(u),
$$


where in $l(u)$ we have collected all lower order terms. Then

$$
\lim _{\left\|u^{\prime}\right\| \rightarrow \infty} \frac{l(u)}{\left\|u^{\prime}\right\|^{2}}=0
$$

Finally, we have

$$
\left(1+b-\left\|h^{\prime}\right\|_{\infty}\right) \leqslant \frac{\pi^{2}}{4} \int_{0}^{\pi} a_{1}^{-}(t) d t+\frac{\pi}{2}\left\|a_{2}\right\|+\frac{l(u)}{\left\|u^{\prime}\right\|^{2}} .
$$

It follows from this and (11) that for some $M \in \mathbf{R}^{+}$we have

$$
\left\|u^{\prime}\right\| \leqslant M
$$

for all solutions of (13). Since $\|u\| \leqslant\left\|u^{\prime}\right\|$, we see that all solutions of (13) are bounded with respect to the $\|\cdot\|_{2,2}$-norm.

It is now easily seen that $\|N u\|_{1}$ is bounded for all solutions of (13). So, if we set $v:=L u$, then $\|v\|_{1}$ is also bounded. Without loss of generality we may assume that $L$ is invertible. Then (13) is equivalent to

$$
v=r N L^{-1} v
$$

We have shown that for some $K \in \mathrm{R}^{+}$we have

$$
\|v\|_{1} \leqslant K
$$

for every solution of (14). Since $N L^{-1}: L^{1}(0, \pi) \rightarrow L^{1}(0, \pi)$ is compact, the assertion follows.

REMARK 3. (a) It should be noted that condition (11) allows some flexibility between $a_{1}(t), a_{2}(t)$ and $h(t)$, since it does not involve the $\|\cdot\|_{\infty}$-norms of $a_{1}$ and $a_{2}$.

(b) Following ideas from [6, Theorem 2.1], it is also possible to allow the nonlinearity $g$ to depend on $u^{\prime \prime}$.

\section{REFERENCES}

[1] A.R. Aftabizadeh, 'Existence and uniqueness theorems for fourth-order boundary value problems', J. Math. Anal. Appl. 116 (1986), 415-426.

[2] R.P. Agarwal, 'On fourth order boundary value problems arising in beam analysis', $D$ ifferential Integral Equations 2 (1989), 91-110.

[3] H. Amann, 'Fixed point equations and nonlinear eigenvalue problems in ordered Banach spaces', SIAM Rev. 18 (1976), 620-709. 
[4] D.G. DeFigueiredo, 'Semilinear elliptic equations at resonance: Higher eigenvalues and unbounded nonlinearities', in Recent Advances in Differential Equations, Editor R. Conti, pp. 89-99 (Academic Press, New York, 1981).

[5] K. Deimling, Nonlinear Functional Analysis (Springer-Verlag, Berlin, Heidelberg, New York, 1985).

[6] C.P. Gupta, 'Existence and uniqueness theorems for the bending of an elastic beam equation', Appl. Anal. 26 (1988), 289-304.

[7] H. Hu, Variational principles of theory of elasticity with applications (Gordon and Breach, New York, 1984).

[8] G. Metzen, 'Existence results of semilinear equations at resonance', (preprint).

[9] M. Protter and H. Weinberger, Maximum Principles in Differential Equations (Prentice-Hall, Englewood Cliffs, N.J., 1967).

[10] R.A. Usmani, 'A uniqueness theorem for a boundary value problem', Proc. Amer. Math. Soc. 77 (1979), 329-335.

[11] K. Washizu, Variational methods in elasticity and plasticity (Pergamon Press, Oxford, 1968).

[12] Y. Yang, 'Fourth-order two-point boundary value problem', Proc. Amer. Math. Soc. 104 (1988), 175-180.

[13] E. Zeidler, Nonlinear Functional Analysis and its Applications I - Fixed-Point Theorems (Springer-Verlag, Berlin, Heidelberg, New York, 1986).

Department of Mathematical Sciences

Memphis State University

Memphis, TN 38152

United States of America 